\title{
Regression of colonic low grade B cell lymphoma of the mucosa associated lymphoid tissue type after eradication of Helicobacter pylori
}

\author{
M Raderer, F Pfeffel, G Pohl, C Mannhalter, J Valencak, A Chott
}

\begin{abstract}
Background-Lymphoma of the mucosa associated lymphoid tissue (MALT) arising in the stomach has been shown to be related to Helicobacter pylori infection, and total regression of gastric lymphoma after successful eradication of $H$ pylori has consistently been reported. MALTtype lymphoma at other localisations, however, has to our knowledge not been linked to $H$ pylori, and eradication of the bacteria has not been studied for management of such lymphomas.
\end{abstract}

Patient/method-A 67 year old man was diagnosed with MALT-type lymphoma simultaneously involving the stomach and the colon descendens. In addition to the presence of MALT-type lymphoma, $H$ pylori associated chronic gastritis was diagnosed, and treatment with clarithromycin, metronidazole, and omeprazole was initiated, resulting in its successful eradication. Results-Follow up performed four months later showed regression of the colonic manifestation, whereas the gastric lymphoma did not respond to antibiotic treatment, as assessed by regular follow up for 14 months, in spite of its restriction to mucosa and submucosa. The patient was therefore treated with oral cyclophosphamide (100 mg a day) resulting in partial remission after seven months of continuous treatment. Because of the presence of residual lymphoma, additional irradiation was performed, which led to complete remission of the gastric lymphoma. The patient remains in complete remission 40 months after diagnosis and 26 months after initiation of treatment.

Conclusion-In the case of concurrent gastric and intestinal low grade MALTtype lymphoma, $H$ pylori eradication may cause regression of the intestinal lesion. (Gut 2000;46:133-135)

Keywords: mucosa associated lymphoid tissue; colon; Helicobacter pylori eradication; lymphoma

The concept of lymphoma of the mucosa associated lymphoid tissue (MALT) type was introduced by Isaacson and Wright in $1983 .{ }^{1}$
MALT-type lymphomas usually arise in organs originally devoid of lymphoid tissue, which is acquired through chronic antigenic stimulation triggered by persistent infections and/or autoimmune processes. ${ }^{2}$ Most MALT-type lymphomas occur in the stomach, but they may arise at any site along the gastrointestinal tract and may also involve extragastrointestinal organs such as the salivary gland, thyroid gland, lung, ocular adnexa, bladder, and skin. ${ }^{3}$ Since the introduction of this clinicopathological entity, MALT-type lymphoma of the stomach has become a focus of interest for both pathologists and clinicians. The discovery of a definitive role of Helicobacter pylori in the pathogenesis of this disease along with the demonstration of complete regression of malignancy after successful eradication of $\mathrm{H} \mathrm{pylori}^{4}{ }^{5}$ has revolutionised treatment options for low grade gastric lymphoma of the MALT type. In a recently published study of 50 patients followed for a median time of two years, complete remission could be obtained after $H$ pylori eradication in about $80 \%$ of patients, ${ }^{6}$ which confirms the results from other series. ${ }^{7-9}$ According to these findings, $H$ pylori eradication seems to be the treatment of choice for patients with low grade gastric MALT type lymphoma. This benefit, however, appears to be restricted to patients with early stages of the disease - that is, involvement of mucosa and submucosa-while more advanced stages as well as patients with high grade disease do not appear to be responsive to removal of the antigenic stimulus provided by $H$ pylori.

Scattered reports in the literature have claimed the propensity of MALT-type lymphoma to occur multifocally involving multiple sites of acquired MALT throughout the human body. ${ }^{10}$ In addition, late relapse of low grade gastric MALT-type lymphoma at gastrointestinal or extragastrointestinal MALT sites such as the lung has repeatedly been reported. ${ }^{11}{ }^{12}$ Lesions arising outside the stomach, however, have not been linked to the presence of $H$ pylori, and eradication of the bacteria has consequently not been tested as a therapeutic measure in such patients. We report the

Abbreviations used in this paper: MALT, mucosa associated lymphoid tissue.

Guertel 18-20, A-1090

Vienna, Austria. 
case of a 67 year old patient with low grade MALT-type lymphoma synchronously involving the stomach and colon, whose colonic lymphoma regressed completely after successful eradication of $H$ pylori, whereas the gastric lymphoma, which was restricted to the mucosa and the submucosa, did not respond to antibiotic treatment.

\section{Case report}

A 67 year old man was referred to a general hospital with a four month history of persistent epigastric complaints refractory to treatment with $\mathrm{H}_{2}$ blocking agents. Upon gastroscopy, a single ulcer ( $3 \mathrm{~cm}$ in diameter) was discovered in the gastric corpus, and multiple biopsy specimens were taken from the ulcer as well as from the surrounding mucosa. Histological evaluation of the biopsy specimens obtained from the ulcer disclosed infiltration with small lymphoid cells invading and destroying the epithelium to form lymphoepithelial lesions. Immunophenotyping showed $\kappa$ light chain restriction, indicating monoclonal $\mathrm{B}$ cell proliferation, and the lymphoid cells stained positive for CD20 and IgM, but were negative for CD 5 and CD10. The proliferation rate was about $10 \%$ as assessed by staining with the MIB1 (Ki 67) antibody. Based on the characteristic histopathological and phenotypic features, the diagnosis of low grade B cell lymphoma of the MALT type was established. In addition to the presence of MALT-type lymphoma, $H$ pylori associated chronic gastritis was diagnosed.

Eradication of $H$ pylor $i$ with clarithromycin $(3 \times 250 \mathrm{mg})$, metronidazole $(2 \times 500 \mathrm{mg})$, and omeprazole $(2 \times 20 \mathrm{mg})$, all given orally for 14 days, was initiated, and the patient was referred to our department for further staging. Staging procedures consisting of ear, nose, and throat examination, endosonography of the upper gastrointestinal tract, enteroclysis, computed tomography scan of the thorax and abdomen, colonoscopy, and a bone marrow biopsy were performed within one week of the initial diagnosis. Endosonography confirmed the presence of a tumorous lesion involving mucosa and submucosa, but no evidence for deeper penetration or local lymph node involvement could be found. Apart from a slight thickening of the gastric wall, no pathological changes within the abdomen and the thorax were seen on computed tomography, and otolaryngological examination, enteroclysis, and bone marrow biopsy were also normal. Upon colonoscopy, however, a sessile polyp measuring about $1.5 \mathrm{~cm}$ was detected in the colon descendens, while no abnormalities were found in the rest of the colon and in the terminal ileum. Multiple biopsy specimens obtained from the colonic polyp disclosed infiltration by low grade B cell lymphoma of the MALT type, which on morphological and immunophenotypical grounds was identical with that in the stomach. Genomic DNA extracted from paraffin embedded tissue from the gastric and the colonic lesion was analysed by polymerase chain reaction for clonal rearrangement of the immunoglobulin heavy chain gene. Simultaneous separation of the two polymerase chain reaction products on $6 \%$ polyacrylamide gels showed one distinct band each at the same position, strongly suggesting the presence of the same monoclonal B cell rearrangement.

As the patient refused any therapeutic intervention, a wait and see strategy with meticulous follow up was adopted.

Follow up investigations were performed four months after initial diagnosis, and consisted of gastroscopy, endosonography, and colonoscopy. Endosonography showed no change in the gastric lymphoma, and histological evaluation of multiple biopsy specimens confirmed the presence of MALT-type lymphoma, but disclosed the absence of $H$ pylori in the stomach with pronounced regression of the inflammatory infiltrate. Colonoscopy, however, showed complete disappearance of the colonic lymphoma both macroscopically and during histological examination of multiple biopsy samples obtained from the former site of the polyp. No response of the gastric lymphoma was shown on re-examination six, nine, and 14 months after initial diagnosis. The patient was therefore treated with oral cyclophosphamide $(100 \mathrm{mg}$ a day) resulting in a partial remission after seven months of continuous treatment, as judged by regular follow up with histological evaluation. Because of the presence of residual lymphoma, radiation of the stomach at a dose of 35 Gy was performed, which led to complete remission of the gastric lymphoma. The patient remains in complete remission 40 months after diagnosis and 26 months after initiation of treatment.

\section{Discussion}

Although MALT-type lymphoma may occur in a wide variety of organs, the stomach is the predominant localisation and constitutes about $70 \%$ of all cases. ${ }^{3}$ According to pioneering work published in the early $1990 \mathrm{~s},{ }^{45}$ infection with $H$ pylori provides the antigenic drive for development of low grade MALT-type lymphoma in the large majority of cases, and transformation to high grade malignancy in the course of the disease is a commonly observed phenomenon. ${ }^{13}$ According to this concept, eradication of $H$ pylor $i$ has been investigated as the first line treatment for patients with MALT-type lymphoma of the stomach, and has been found to be highly effective in patients with localised low grade disease. ${ }^{14}$ To date, patients with more disseminated disease or those with high grade malignancy are generally not thought to be responsive to antibiotic treatment alone. Although a common consensus has been reached on these issues, there is hardly any evidence to suggest eradication of $H$ pylori for treatment of patients with MALTtype lymphoma located outside the stomach.

In our patient, total regression of a colonic manifestation was achieved after successful eradication of $H$ pylori four months after initiation of antibiotic treatment. Regular follow up by colonoscopy, performed every six months for the first two years and once a year thereafter, has so far not detected any evidence of disease recurrence in the colon three years after $H$ 
pylori eradication. Given the concept underlying the development of gastric MALT-type lymphoma, one would assume an $H$ pylori associated gastric lymphoma as the primary focus of disease in our patient, with secondary involvement of the colon. That this sequential order of dissemination is indeed the case has recently been confirmed by Du et al. ${ }^{15}$ Our histological, phenotypic, and genetic studies strongly suggest that the concurrent gastric and colonic lymphomas were derived from the same clone. Because of the postulated common mucosal immunity, an initially $H$ pylor $i$ associated lymphoma could theoretically remain dependent on $H$ pylori associated $\mathrm{T}$ cells for some time even after generalisation, which further underscores the potential gastric origin of the lesions encountered. Of interest, however, is the fact that the gastric lymphoma did not respond within a follow up period of 14 months, as confirmed by regular endosonographic and gastroscopic follow up with histological examination. One may speculate, however, that, with a longer follow up, a response in the gastric lesion would also have been observed. Nevertheless, this appears to be highly unlikely; although responses to successful $H$ pylori eradication have been observed after a prolonged period of time in some studies, the median time to remission in the most comprehensive series published so far was 5.5 months, and all responding patients showed at least some signs of regression endoscopically or histologically at an earlier time, ${ }^{6}$ which was clearly not the case in our patient. Nevertheless, we cannot offer a definite explanation for the dissociation of responses between the two localisations of the lymphoma. One hypothetical explanation is the presence of a high grade lymphoma component undetected by multiple forceps biopsies, as has been reported for patients with gastric MALT-type lymphoma not responding to $H$ pylori eradication. ${ }^{6}$ In the case of high grade transformation, however, one would expect at least some progression of the disease over the relatively long time of 14 months. Alternatively, responsiveness to $H$ pylori eradication has been shown to be an indicator for clonal evolution of the disease, as $H$ pylori induced stimuli appear necessary only at an early stage of malignancy; once genetic damage has accumulated to a certain "point of no return", eradication of $H$ pylori does not cause a clinical response. ${ }^{6}$ In this context, one may postulate dissemination of $H$ pylori dependent gastric lymphoma to the colon at an early stage, with additional as yet undiscovered factors causing faster evolution in the gastric lesion as compared with the still responsive colonic lymphoma.

This report shows that, in the case of concurrent gastric and intestinal low grade MALT-type lymphoma, $H$ pylori eradication may cause regression of the intestinal lesion.

1 Isaacson PG, Wright DH. Malignant lymphoma of mucosaassociated lymphoid tissue. A distinctive type of B-cell associated lymphoid tissue. A distinct
lymphoma. Cancer 1983;52:1410-16.

2 Greiner A, Marx A, Heesemann J, et al. Idiotype idendity in MALT-type lyphoma and B-cells in Helcobacter-pyloriassociated chronic gastritis. Lab Invest 1994;70:572-8.

3 Zucca E, Roggera E, Bertoni F, et al. Primary extranodal non-Hodgkin's lymphomas. Part 1: gastrointestinal, cutaneous and genitourinary lymphomas. Ann Oncol 1997;8: 727-37.

4 Wotherspoon AC, Ortiz-Hidalgo C, Falzon MR, et al. Helicobacter pylori-associated gastritis and primary B-cell gastric lymphoma. Lancet 1991;338:1175-6.

5 Wotherspoon AC, Doglioni C, Diss TC, et al. Regression of

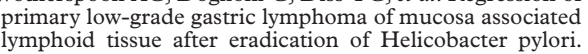
Lancet 1993;342:575-7.

6 Neubauer A, Thiede C, Morgner A, et al. Cure of Helicobacter pylori infection and duration of remission of low-grade gastric mucosa-associated lymphoid tissue ymphoma. $\mathscr{f}$ Natl Cancer Inst 1997;89:1350-5.

7 Bayerdörffer E, Neubauer A, Rudolph B, et al. Regression of primary gastric lymphoma of mucosa associated lymphoid tissue type after cure of Helicobacter pylori infection. Lancet 1995;345:1591-4.

8 Roggero E, Zucca E, Pinotti G, et al. Eradication of Helicobacter pylori infection in primary low-grade gastric ymphoma of the mucosa-associated lymphoid tissue. Ann Intern Med 1995;122:767-9.

9 Montalban C, Manzanal A, Boixeda D, et al. Helicobacter pylori eradication for the treatment of low-grade gastric MALT-lymphoma: follow-up together with sequential molecular studies. Ann Oncol 1997;8:37-40.

10 Graziadei G, Pruneri G, Carboni N, et al. Low-grade MALT-lymphoma involving multiple mucosal sites and MALT-lymphoma involving multiple muco
bone marrow. Ann Hematol 1998;76:81-3.

11 Stephen MR, Farquharson MA, Sharp RA, et al. Sequential MALT lymphomas of the stomach, small intestine and gall bladder. F Clin Pathol 1998;51:77-9.

12 Kawamata N, Miki T, Fukuda T, et al. Determination of a common clonal origin of gastric and pulmonary mucosaassociated lymphoid tissue lymphomas presenting five years apart. Intern Med 1995;34:220-3.

13 Chan JK, Ng CS, Isaacson PG. Relationship between highgrade lymphoma and low-grade B-cell mucosa-associated lymphoid tissue lymphoma (MALT lymphoma) of the stomach. Am F Pathol 1990;136:1153-64.

14 Thiede C, Morgner A, Alpen B, et al. What role does Helicobacter pylori eradication play in gastric MALT and gastric MALT lymphoma. Gastroenterology 1997;113(suppl 6):S61-4.

$15 \mathrm{Du} \mathrm{M}, \mathrm{Xu} \mathrm{C}, \mathrm{Diss} \mathrm{T}$, et al. Intestinal dissemination of gastric mucosa-associated lymphoid tissue lymphoma. Blood 1996;88:4445-51. 